\title{
Parton-shower matching systematics in vector-boson-fusion WW production
}

\author{
Michael Rauch $^{1, a}$, Simon Plätzer ${ }^{2,3}$ \\ ${ }^{1}$ Institute for Theoretical Physics, Karlsruhe Institute of Technology, Karlsruhe, Germany \\ ${ }^{2}$ Institute for Particle Physics Phenomenology, Durham University, Durham, UK \\ ${ }^{3}$ School of Physics and Astronomy, University of Manchester, Manchester, UK
}

Received: 6 June 2016 / Accepted: 26 April 2017 / Published online: 8 May 2017

(C) The Author(s) 2017. This article is an open access publication

\begin{abstract}
We perform a detailed analysis of next-to-leading order plus parton-shower matching in vector-boson-fusion WW production including leptonic decays. The study is performed in the Herwig 7 framework interfaced to VBFNLO 3 , using the angular-ordered and dipole-based parton-shower algorithms combined with the subtractive and multiplicativematching algorithms.
\end{abstract}

\section{Introduction}

Vector-boson fusion (VBF) and vector-boson scattering (VBS) belong to an important class of processes for the Large Hadron Collider (LHC) at CERN. These processes are characterized by the electroweak production of single bosons and di-bosons in association with two jets, respectively, where a quark or anti-quark scatters with another quark or anti-quark through the space-like exchange of an electroweak boson. VBF and VBS production are particularly important for the recently started run-II phase of the LHC, as their cross sections significantly increase due to the higher centre-of-mass energy of $13 \mathrm{TeV}$. Its study was first suggested for the VBF production of Higgs bosons [1-10]. In the following, to simplify notation we will collectively refer to both types of processes as VBF.

The characteristic feature of the VBF class of processes are two energetic jets in the forward regions of the detector, the so-called tagging jets [11]. In the central region, only a low jet activity is observed. The leptonic decay products of the vector bosons are typically found between the two tagging jets. These properties allow us to distinguish VBF from two types of background processes with the same final state. At the same order in the coupling constants, di-boson or triboson production, where one of the bosons decays hadronically, contributes [12-21]. For these processes, the invariant

a e-mail: michael.rauch@kit.edu mass of the two jets is close to the mass of the decaying boson, and larger values are strongly suppressed. The other class of irreducible backgrounds is QCD-induced production in association with two jets [22-36]. There, the two jets are preferably emitted in the central region. As two powers of the electromagnetic coupling constant get replaced by their strong counterpart, and for most boson combinations gluoninduced production channels are also possible, this production mode will dominate for inclusive cross sections. Applying tight VBF cuts allows us to reduce these background processes and suppress any interference effects [29]. These cuts typically require a large invariant mass of the two tagging jets of the order of several hundreds $\mathrm{GeV}$, and a large rapidity separation between them. They also reduce any interference effects between $t$ - and $u$-channel exchange diagrams to a completely negligible level and justify the often used socalled VBF approximation or structure-function approach, where these contributions are not taken into account. A veto on additional jets [37] can further enhance the signal-tobackground ratio.

The appearance of triple and, in the case of di-boson production, quartic gauge-boson vertices makes VBF processes an ideal tool for studying these. In the high-energy region, a strong cancellation between diagrams with quartic vertices, triple vertices, and Higgs boson exchange takes place. Any modifications of the couplings from their Standard Model (SM) values could spoil this cancellation and lead to a rise of the squared matrix element proportional up to the eighth power of the di-boson invariant mass. VBF is therefore a sensitive probe of these anomalous contributions to the SM gauge couplings. Also, the existence of additional heavy Higgs bosons from additional singlets or doublets can be probed in the di-boson invariant-mass distribution $[10,38]$.

To investigate such effects of physics beyond the Standard Model (BSM), a precise knowledge of the underlying SM prediction is necessary. Next-to-leading order (NLO) QCD 
corrections to all VBF processes have been computed [3946]. Their effect is typically rather modest, of the order of $10 \%$ or below. Choosing the momentum transfer through the space-like bosons as a scale choice has been proven to be a very good choice. A dedicated implementation of all VBF processes at NLO QCD accuracy, including leptonic decays of the vector bosons and the option to switch on anomalous coupling effects or some BSM models such as a two-Higgs model, is available in the VBFNLO program [47-50].

The combination of NLO QCD results with parton showers has been studied thus far for some of the VBF processes [25,51-56] using the Powheg-Box framework [5759]. Additional corrections have so far been calculated only for VBF- $H$ production. These are the NLO electroweak corrections [60-62], which turn out to be of a similar size to the NLO QCD ones. For the measured Higgs mass they are of opposite sign for inclusive cross sections. Also known are the NLO QCD corrections for VBF- $H$ production in association with three jets $[63,64]$. A third type are the next-tonext-to-leading order QCD corrections. While corrections to the inclusive cross section, calculated in the structurefunction approach, are well below the percent level $[65,66]$, there are much larger effects when considering differential distributions in the VBF approximation [67]. These are, however, mostly due to the additional effects from double realradiation processes. Adding parton-shower effects on top of NLO QCD results in general improves the agreement, though relevant differences in some distributions remain.

For a detailed understanding of VBF processes the matching of NLO QCD predictions with parton showers is therefore necessary. This includes not only the central predictions, but also trying to quantify the associated theory errors. Tools to assess them are for example the variation of various scales appearing in the predictions. However, one can also compare different matching and parton-shower algorithms. Combining the fast and accurate predictions of VBFNLO with the flexible options of Herwig 7 hence offers us unique possibilities to study these effects. As it is important to have control of these uncertainties in the perturbative part of the simulation, we will not consider any effects due to hadronization or multiple parton interactions. These are left for a future publication.

\section{Outline of the simulation}

\subsection{NLO and NLO+PS matching with Herwig 7}

The newly released Herwig 7 Monte Carlo event generator $[68,69]$ builds on its successful predecessor Herwig++ [70]. It features significantly improved physics capabilities, particularly for NLO QCD corrections and their combination with the two available parton-shower modules based on Refs. [71] and [72].
Based on extensions of the previously developed Matchbox module [73], NLO event simulation can be carried out with the help of external amplitude providers, which are used by Herwig to evaluate tree-level and one-loop matrix elements. These are then automatically combined with the Catani-Seymour dipole subtraction [74,75], and generalpurpose as well as specialized phase-space generation algorithms to assemble a full NLO calculation. This NLO calculation can be further extended by the automatically determined matching subtractions to combine it with a downstream parton-shower algorithm. While a number of hard process calculations are supported by dedicated Matchbox plugins, communication with external general-purpose amplitude providers, VBFNLO $3[47,50,76]$ in the context of this study, takes place via extensions of the BLHA 2 standard [77].

NLO predictions obtained from the Herwig+VBFNLO setup have extensively been validated against standalone calculations obtained from VBFNLO, using both a range of integrator and phase-space generation algorithms either supplied by the standard Matchbox modules or employing the versatile interface structure to use the according VBFNLO routines. Extensive comparisons, also to experimental data, for a host of processes using the Rivet framework [78] are available on the Herwig homepage [79] and in Ref. [80].

\subsection{VBFNLO 3}

VBFNLO $[47,50,76]$ is a flexible parton-level Monte Carlo generator for processes with electroweak bosons. It provides a fully differential simulation of VBF processes, amongst others, at NLO QCD accuracy.

The communication with Herwig is done via an interface based on the BLHA 2 standard [77]. The VBFNLO implementation contains some extensions on top of the standard to access additional features such as the internal phasespace generator, which has been used for the results presented in this article. None of them is mandatory, however, and a standards-compliant Monte Carlo generator is sufficient to obtain amplitudes from VBFNLO.

In this article, we are considering as an example the electroweak production process

$$
p p \rightarrow W^{+} W^{-} j j \rightarrow e^{+} v_{e} \mu^{-} \bar{v}_{\mu} j j .
$$

We include the leptonic decays of the $W$ bosons including full off-shell effects. Contributions from non-resonant diagrams as well as those with Higgs bosons are included. The latter are not only important in phase-space regions where the Higgs boson becomes on-shell, but also at large invariant masses of the four leptons, where a significant cancellation between Higgs boson and continuum diagrams takes place $[10,38]$. For the partons we restrict ourselves to the VBF approximation, where interference effects between same-flavour quarks 

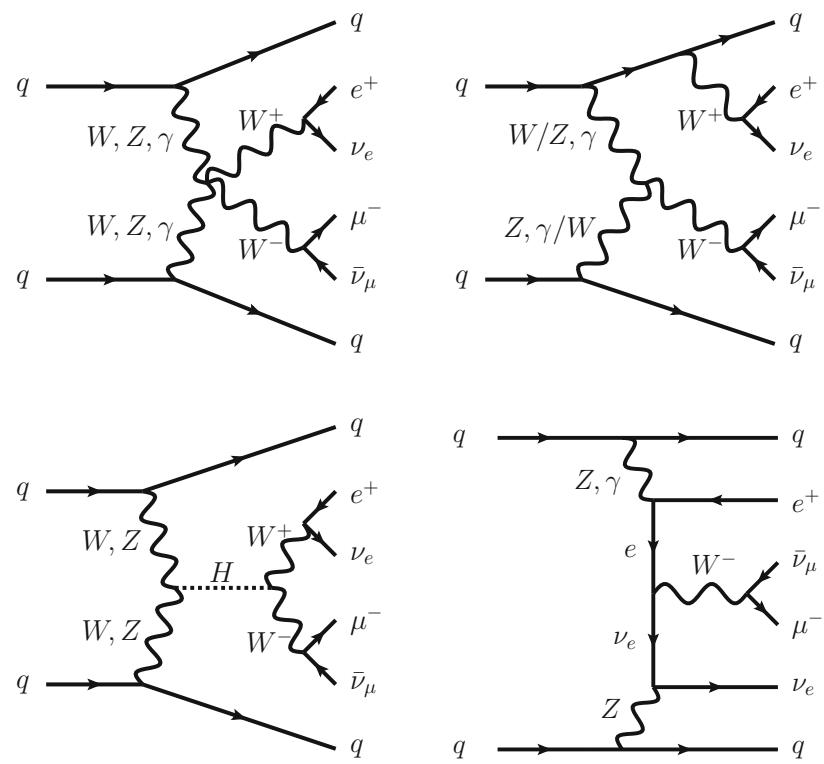

Fig. 1 Example Feynman diagrams for $\mathrm{VBF}-W^{+} W^{-} j j$ production at LO including leptonic decays and non-resonant contributions

in the final-state are neglected. These terms are both phasespace and colour suppressed. When imposing VBF-specific cuts, their contribution to the cross section becomes negligible. Some example Feynman diagrams of the LO process are depicted in Fig. 1. They show the rich structure of this process, which includes contributions from quartic gaugeboson vertices (top left diagram), triple gauge-boson vertices (top right), and Higgs-mediated exchange (bottom left). An example of a non-resonant contribution is shown in the bottom right diagram of Fig. 1. The NLO QCD corrections to this process have been first presented in Ref. [41].

\section{Matching algorithms and uncertainties}

Matchbox currently supports direct, subtractive matching (i.e. MC@NLO-type [81]) to both the angular ordered and dipole showers, as well as multiplicative (i.e. Powheg-type [57]) matching. Conceptually, as well as technically, these algorithms are calculating matched cross sections as ${ }^{1}$

$$
\begin{aligned}
\sigma_{\mathrm{NLO}}^{\text {matched }}= & \int_{n}\left(\mathrm{~d} \sigma_{\mathrm{LO}}+\mathrm{d} \sigma_{\mathrm{virt}}\right) \\
& +\int_{n} \int_{1}\left(\mathrm{~d} \sigma_{\mathrm{PS}}-\mathrm{d} \sigma_{\mathrm{sub}}\right) \\
& +\int_{n+1}\left(\mathrm{~d} \sigma_{\mathrm{R}}-\mathrm{d} \sigma_{\mathrm{PS}}\right)
\end{aligned}
$$

\footnotetext{
${ }^{1}$ Further details to the matching and the other algorithms provided by Herwig will be subject to an extensive discussion in an upcoming review.
}

where in this schematic notation 'LO' denotes the leading order cross section, 'virt' the contribution by one-loop diagrams, integrated subtraction terms and collinear counterterms, 'sub' denotes un-integrated subtraction terms, ' $R$ ' the real emission and 'PS' is the parton-shower approximation to the real-emission cross section. The second integral in the middle line is performed over the one-particle phase space of the extra emission. In this notation, the parton-shower approximation can also be given by a matrix-element correction [82,83], giving rise to multiplicative, or Powhegtype, matching. Matchbox samples the matrix-element corrections using adaptive methods [84] and is able, for the case of the angular-ordered shower, to add truncated showers on top of it to fully account for large-angle, soft emissions.

Uncertainties are explored by varying the relevant scales in the hard process and showers as outlined in Ref. [85]. For both of the showers, as well as for the matrix-element correction entering the multiplicative matching, we choose to use the resummation profile scale [85] to guarantee a smooth transition between the hard matching and resummation regions, while maintaining the resummation properties of the parton shower.

\section{Phenomenological results}

We perform parton-level studies, treating all partons as massless. As we are not interested in effects from top-quark production, we apply a veto on any bottom quarks appearing in the final state. Multiple parton interactions (MPI) are not included, and showering is performed using both Herwig shower modules at their default settings. We also employ default settings for the hard process calculations, including the MMHT2014 PDF set [86] with five active flavours.

We apply typical VBF selection cuts,

$$
\begin{array}{rlrl}
p_{T, j} & >30 \mathrm{GeV}, & \left|y_{j}\right|<4.5, \\
p_{T, \ell} & >20 \mathrm{GeV}, & \left|y_{\ell}\right| & <2.5, \\
m_{e^{+}, \mu^{-}} & >15 \mathrm{GeV}, & \\
m_{j 1, j 2} & >600 \mathrm{GeV}, & \left|y_{j 1}-y_{j 2}\right| & >3.6,
\end{array}
$$

and consider $p p$ collisions at a centre-of-mass energy of $13 \mathrm{TeV}$. Jets are clustered from partons using the anti- $k_{T}$ algorithm [87] with a cone radius of $R=0.4$. The choice of cuts is adopted from the cut-based VBF category of the $H \rightarrow W W$ study of ATLAS [88]. The corresponding CMS analysis [89] uses very similar values. We take the transverse momentum of the leading jet, $\mu_{0}=p_{T, j 1}$, as the central scale choice. This selection is useful both from the fixed-order part of the calculation and the parton-shower one. In the VBF region, where the tagging jets are high-energetic and emitted under a small scattering angle, their transverse momentum 


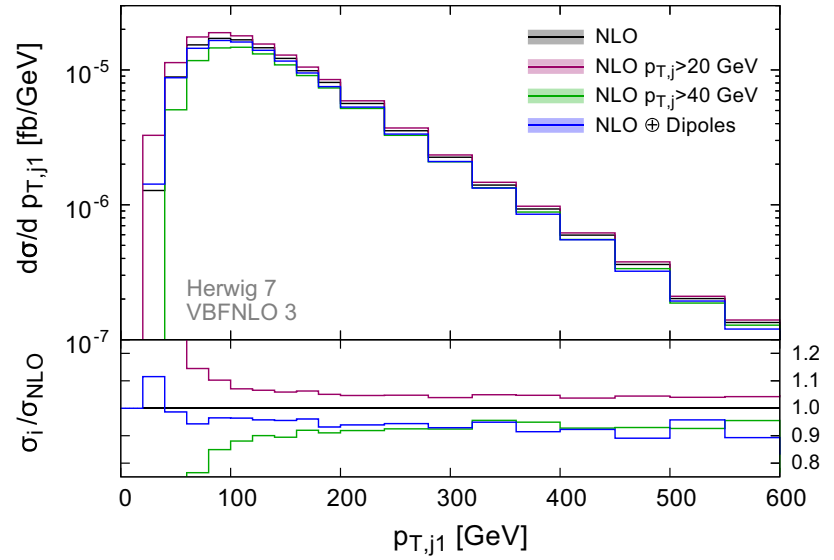

Fig. 2 The transverse-momentum spectrum of the leading jet for a number of different cuts on the fixed-order calculation comparing the central NLO and showered result using a transverse-momentum threshold of $30 \mathrm{GeV}$. Cut migration effects for the central predictions at high transverse momenta are at the level of $10 \%$, while they can have a significant impact in lower- $p_{\perp}$ observables

is approximately equal to the absolute value of the momentum transfer via the exchanged vector boson, which has been shown to be an advantageous scale choice [43]. For the parton shower, this also defines the hard veto scale, the maximal transverse momentum at which the parton shower can generate extra emissions. Setting this to the transverse momentum of the leading jet is consistent with our picture of partonshower evolution.

Processes with jets at the level of the hard process require selection cuts on the jets; additional parton-shower emissions off these jets will migrate contributions across the cut boundary such that jet cross sections after applying parton showering will typically be lower than the input cross section at the level of the hard process. We quantify this effect in Fig. 2 by sliding the jet cut at the level of the fixed-order NLO cross section from $p_{\perp} \geq 20,30$ through $40 \mathrm{GeV}$ and comparing with the showered result using a jet $p_{\perp}$ threshold of $30 \mathrm{GeV}$. As representative observable we take the $p_{\perp}$ spectrum of the leading jet in this case, though similar findings apply to the other observables and inclusive cross sections, as well. The comparison of the parton-shower curve with the different fixed-order ones shows that the differential cross section can be reconciled by increasing the transverse-momentum cut of the fixed-order simulation. This can easily be understood, as the additional radiation generated by the parton shower can be sufficiently wide-angle, so that it is no longer combined back into the same jet by the jet clustering algorithm. Thus a larger starting value is required to obtain the same final value.

Choosing the analysis cut to be equal to the generation cut is well contained within the variation of the cut applied at the hard process. We therefore conclude that no further tuning of acceptance criteria to minimize cut migration is required in this study. To err on the side of caution, we nevertheless apply

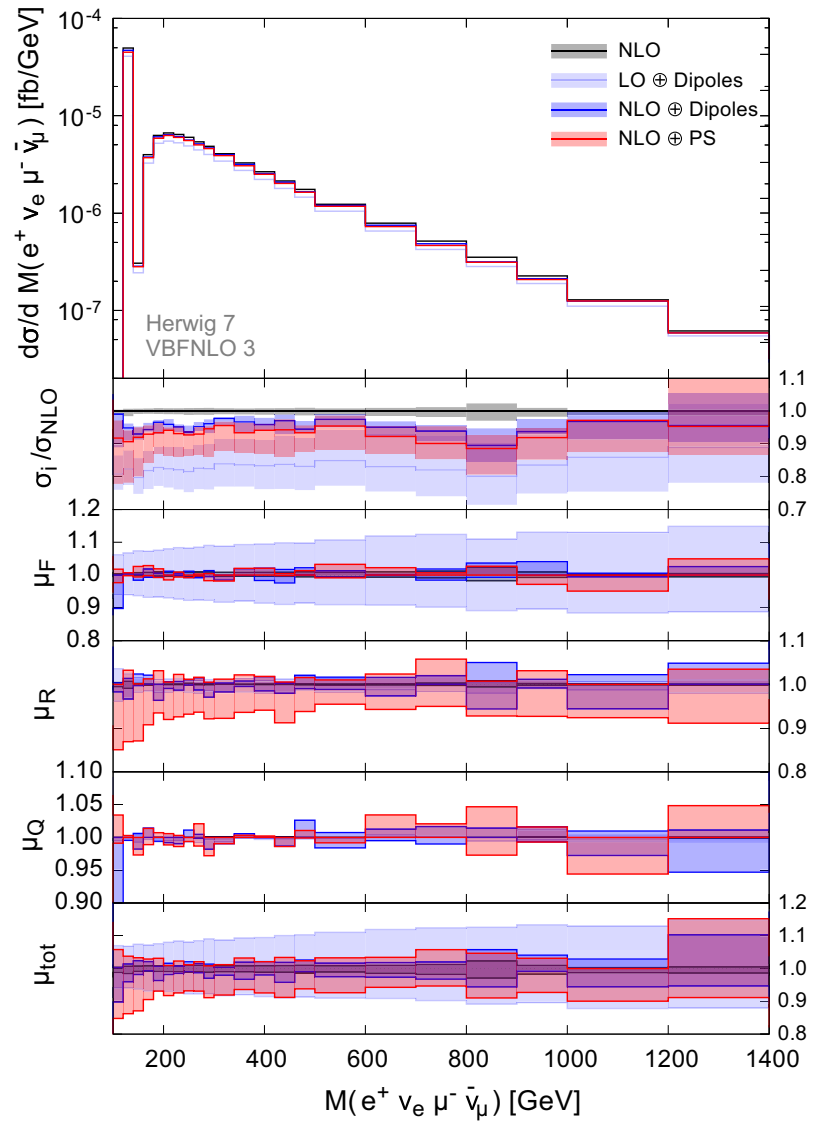

Fig. 3 The invariant 4-lepton mass comparing parton-level NLO results (black) with scale variations, leading order plus dipole shower predictions (light blue), and NLO matched results for the dipole and angular-ordered shower (dark blue and red, respectively). The top ratio plot shows the central predictions and overall variation envelopes with respect to the parton-level fixed-order result; the subsequent ratio plots show the variations of the individual scales with respect to their central predictions, focusing on factorization $\left(\mu_{\mathrm{F}}\right)$, renormalization $\left(\mu_{\mathrm{R}}\right)$ and hard veto scale $\left(\mu_{\mathrm{Q}}\right)$ variations, as well as the overall envelope $\left(\mu_{\text {tot }}\right)$

generation-level cuts which are looser than the ones given in Eq. 3. An event is selected for further processing if at least two jets with transverse momenta larger than $20 \mathrm{GeV}$ within a rapidity range of $|y|<5$ are present, and the two leading jets have an invariant mass of at least $400 \mathrm{GeV}$ with a rapidity separation larger than 3 . Also the lepton cuts are relaxed to a minimum transverse momentum of $15 \mathrm{GeV}$ and an absolute value of the rapidity smaller than 3 . These generation-level cuts help to improve the efficiency of the simulation, as less events are generated which later fail the analysis cuts. We have verified that their impact is compatible with the statistical uncertainties on our simulation.

Contrary to the study presented in Ref. [85], here we have considered the parton showers at their (tuned) default settings rather than the baseline settings; we expect the effects caused by these differences to be small. The only noticeable difference in variations is a larger down-variation of 


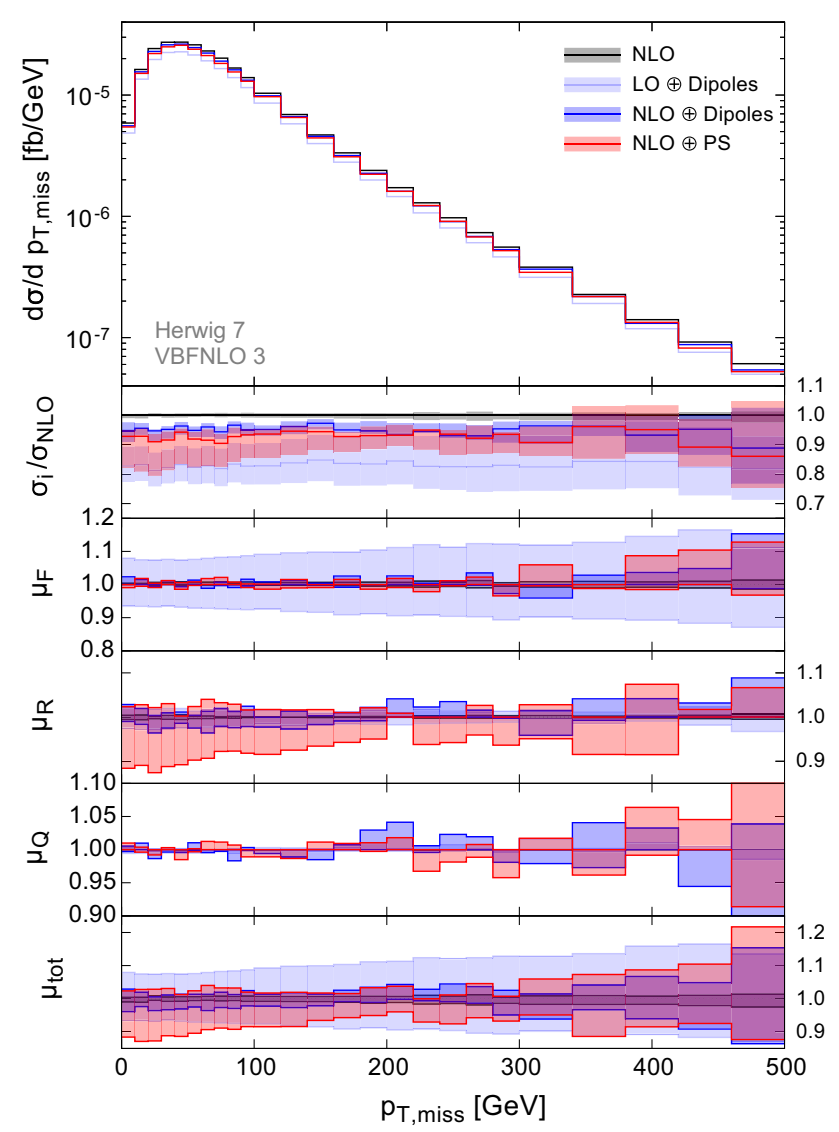

Fig. 4 The cross section predictions differential in the missing transverse momentum. See Fig. 3 and the text for more discussion

the angular-ordered shower when lowering the renormalization scale appearing as argument of the strong coupling; this effect is only visible at the level of the hard tagging jets and we therefore conclude that it is originating from an increased cut migration due to enhanced radiation present in this variation.

Turning to uncertainties we first consider the distribution of the four-lepton invariant mass depicted in Fig. 3. Due to the presence of two neutrinos, this distribution is not directly accessible experimentally, but would have to be replaced by some transverse-mass definition. We nevertheless choose to show it here because of its importance from a theory aspect. The larger upper panel shows the differential distributions using the central scale choice, exhibiting the Higgs boson peak at $125 \mathrm{GeV}$ and the continuum production region above $2 M_{W}$. Curves shown are the parton-level NLO results (black), leading order plus dipole shower (light blue) and NLO matched results for the dipole and angular-ordered shower (dark blue and red, respectively). The uppermost of the smaller panels shows the ratio of the cross section with respect to the parton-level fixed-order result, while the bands depict the overall scale variation envelopes. The four lower panels show the changes of the differential cross section when varying, from top to bottom, the factorization $\left(\mu_{\mathrm{F}}\right)$, renormal-

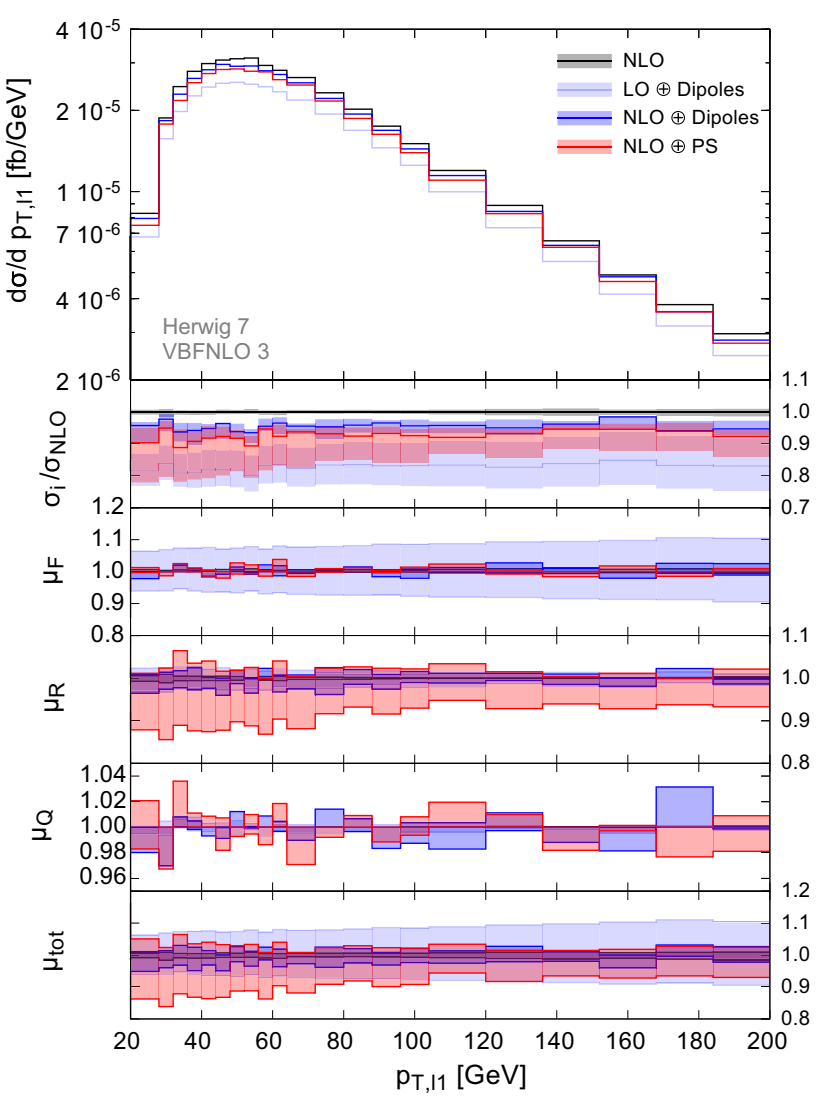

Fig. 5 Same as Fig. 3, showing the $p_{\perp}$ spectrum of the leading charged lepton. The spectrum of the subleading lepton shows a similar behaviour

ization $\left(\mu_{\mathrm{R}}\right)$ and hard veto scale $\left(\mu_{\mathrm{Q}}\right)$, and all of them $\left(\mu_{\text {tot }}\right)$. Variations are performed in the range $\mu_{i} / \mu_{0} \in\left[\frac{1}{2} ; 2\right]$. For the total uncertainty envelope, we allow the individual scales to vary independently, but require that ratios of scales also fulfill the condition $\mu_{i} / \mu_{j} \in\left[\frac{1}{2} ; 2\right]$.

We find that parton showering only mildly affects the shape of the four-lepton invariant-mass distribution, while the overall normalization is subject to configurations showered 'out' of the VBF acceptance criteria. The shower uncertainties are clearly reduced in changing from LO+PS to NLO+PS simulation, with both showers yielding comparable results both in their central prediction as well as variations. The central predictions can also be compared to the Powheg-Box implementation presented in Ref. [54] using Pythia 6 [90] as parton shower. While the exact values of parameters and cuts differ, they are sufficiently similar for the general features to be expected to be similar. An important difference is that these results contain also effects from hadronization, underlying event and multi-parton interactions. Considering the transverse-mass distribution of the four leptons, as for the invariant-mass distribution shown here hardly any shape changes occur when going from a fixed-order calculation to one matched to parton showers. Similar conclusions apply to other observables probing mainly the electroweak part of the 


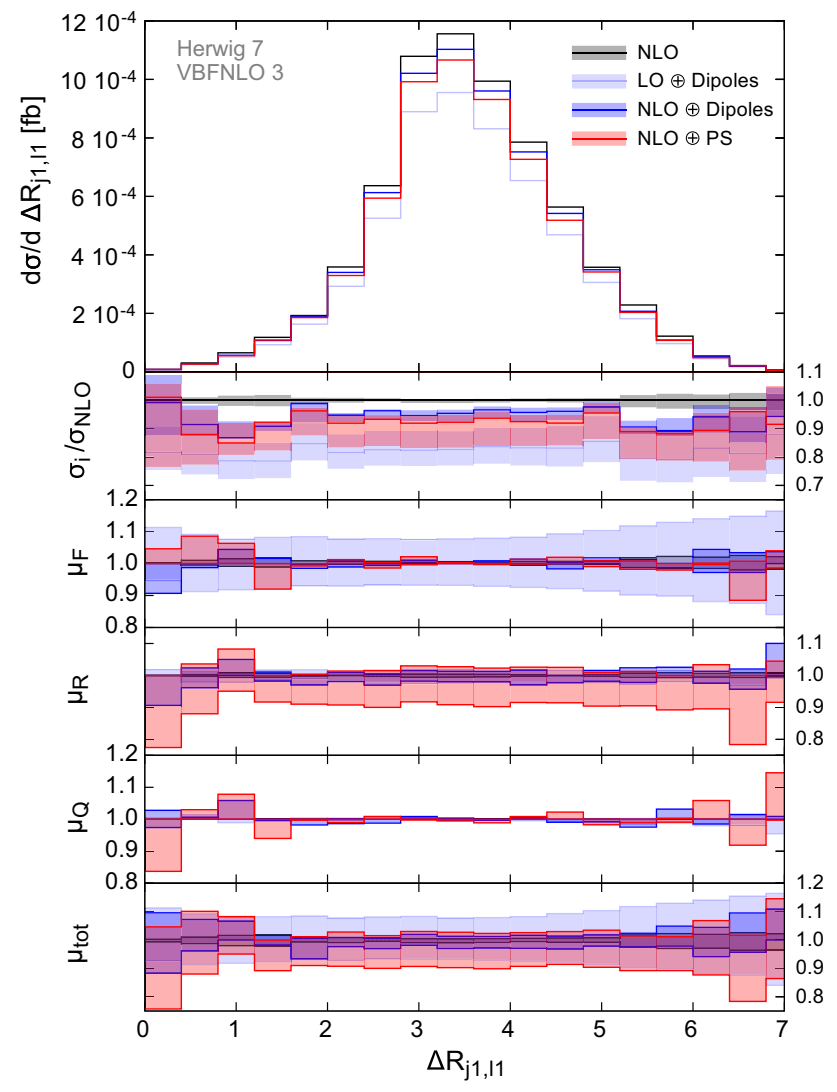

Fig. 6 Same as Fig. 3, showing the separation of the leading charged lepton and the leading jet. Separations with respect to the subleading, second and third, jets show similar features, with increased uncertainties for the third jet as the accuracy of its description is lower by one order

final state, such as the missing transverse-momentum distribution Fig. 4 and the $p_{\perp}$ spectrum of the leading charged lepton Fig. 5.

Further observables required to reconstruct the VBF signature are significantly more affected by parton-shower effects, exemplified here in the case of the separation between the leading lepton and the leading jet shown in Fig. 6, with the separation from the third jet being most sensitive to shower effects; within uncertainties, the showers do, however, yield comparable results.

We finally turn to details of the third jet, as relevant to applying central jet vetoes to suppress the impact of QCDinduced contributions. Since this jet is present at leading order only in the matched simulation and solely consists of parton-shower radiation for the LO+PS setting, larger uncertainties and impact of showering are expected. While small transverse momenta of the third jet are, at NLO+PS, mostly stable with respect to shower effects, Fig. 7, further details of the radiation pattern, particularly the relative position of the third jet with respect to the tagging jets, ${ }^{2}$ Fig. 8 , are sig-

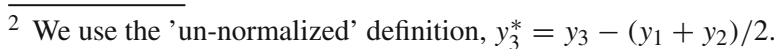

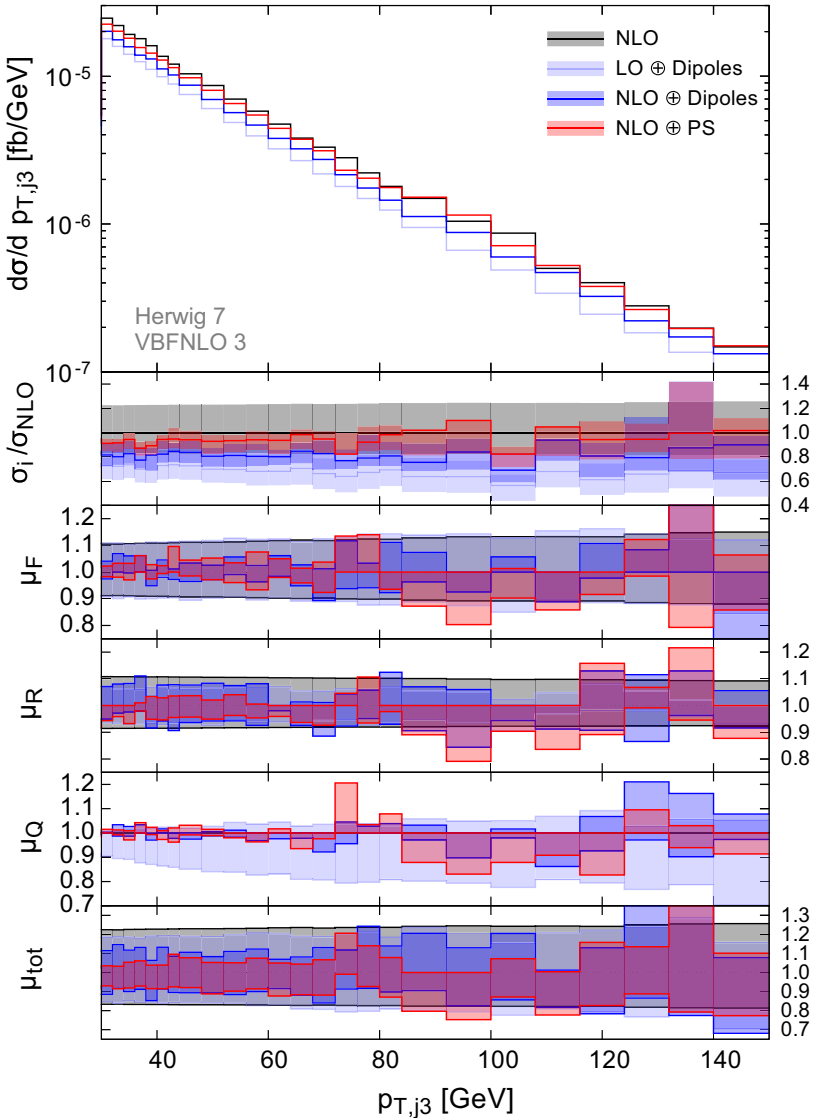

Fig. 7 The differential cross section for the transverse momentum of the third leading jet. See Fig. 3 and the text for more details

nificantly affected by both the impact of NLO versus $\mathrm{LO}$ and the additional shower emissions.

At leading order we observe, for these observables, a large dependence on the shower hard scale $\mu_{\mathrm{Q}}$, which is reduced in the matched simulation though still showing a deviation from the next-to-leading order shape for very central jets in between the tagging jets. One would therefore be worried about the choice of matching scheme, however, using a multiplicative (Powheg-type) matching with a reasonable restriction on the exponentiated phase space by applying the resummation profile scale, we find results compatible with the subtractive matching, cf. Fig. 9. Looking at the same distribution shown in Ref. [54] in the Powheg-Box+Pythia framework, we see that also there the central rapidity gap is preserved once the same transverse-momentum cut on the third jet as on the two tagging jets is imposed. For a LO plus parton-shower setup (see e.g. Ref. [91]), Pythia tends to predict a strong enhancement in the central region and a reduction forward of the tagging jets, while the situation is different for Herwig. Moving to the NLO setup, the contributions get corrected by the real-emission matrix element and move much closer, though some difference still exhibiting this tendency remains. 


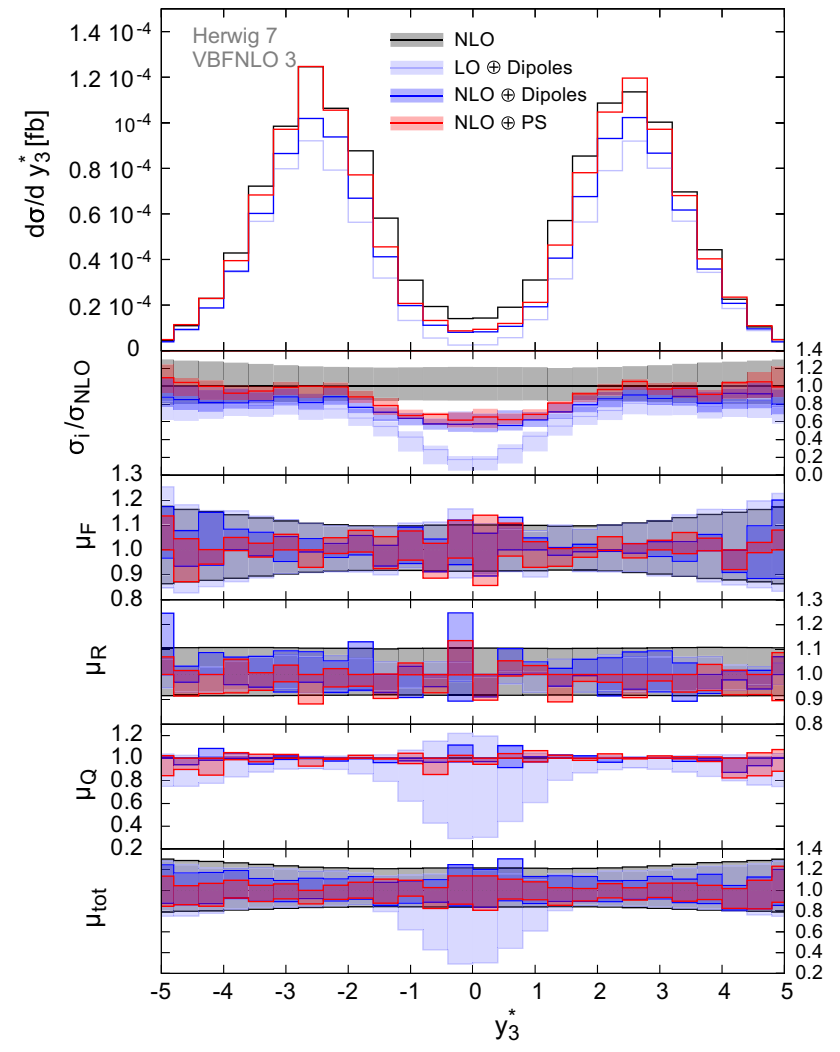

Fig. 8 The relative rapidity of the third leading jet with respect to the tagging jets. See Fig. 3 and the text for more details

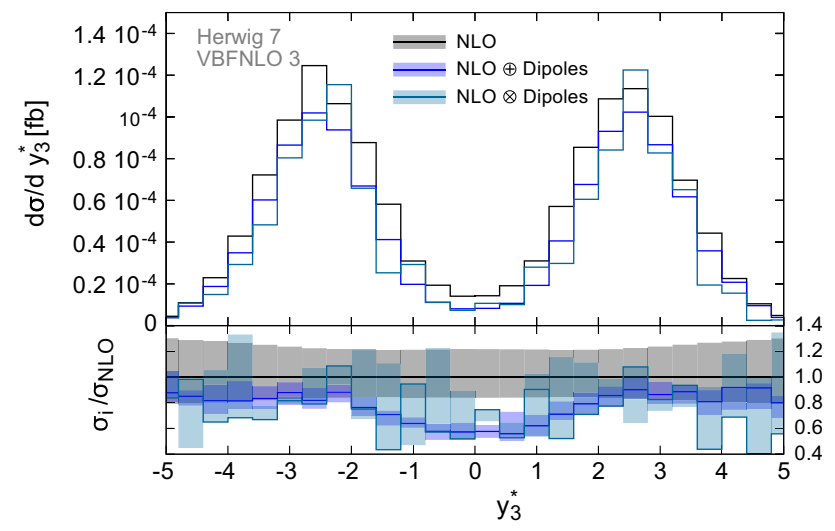

Fig. 9 The relative rapidity of the third jet with respect to the two tagging jets, comparing the fixed-order parton level result (black) to the results obtained with the subtractive (blue) and multiplicative (dark cyan) matching algorithms. The ratio plot shows the ratio with respect to the fixed-order result. The band denotes the change of the differential cross section when varying the factorization, renormalization and hard veto scale jointly around the central value, $\mu_{\mathrm{F}} / \mu_{0}=\mu_{\mathrm{R}} / \mu_{0}=$ $\mu_{\mathrm{Q}} / \mu_{0} \in\left[\frac{1}{2} ; 2\right]$

\section{Conclusions and outlook}

We have presented a study of NLO QCD predictions for electroweak $W W$ plus two-jet production including leptonic decays, off-shell effects and non-resonant contributions. The fixed-order results have been matched to subsequent parton showering using the two shower modules and the Matchbox framework of Herwig 7, which has also been used to obtain the fixed-order results using amplitudes which have been made available via an extended BLHA interface included in VBFNLO 3.

Concentrating on perturbative physics at parton level, we find that matching and parton-shower uncertainties are well under control for this process. Given that the third jet is described only at leading order, and higher jet multiplicities are solely obtained from parton-shower radiation, we argue that multi-jet merging in this case is desirable to further reduce the uncertainties. Cut migration effects seem to impact the predictions at least at the level of $10 \%$ and so require further investigation by e.g. using vanishing generation cuts on jets and applying a reweighting procedure to obtain sufficient statistics within the acceptance of the analysis.

As opposed to uncertainties at the level of the hard process and parton showering, no consistent prescription has yet been obtained to assign uncertainties to the overall event generator prediction including hadronization and multiple partonic interactions (MPI), which we leave for a future study. The present work and tools used in it also constitute an important contribution to a comprehensive programme of employing precision QCD event generators for Higgs phenomenology in the VBF channel.

Acknowledgements We are grateful to the other members of the Herwig and VBFNLO collaborations for encouragement and helpful discussions; in particular we would like to thank Johannes Bellm, Stefan Gieseke and Peter Richardson for a careful review of the manuscript. SP acknowledges support by a FP7 Marie Curie Intra European Fellowship under Grant Agreement PIEF-GA-2013-628739, and the kind hospitality of KIT on various occasions.

Open Access This article is distributed under the terms of the Creative Commons Attribution 4.0 International License (http://creativecomm ons.org/licenses/by/4.0/), which permits unrestricted use, distribution, and reproduction in any medium, provided you give appropriate credit to the original author(s) and the source, provide a link to the Creative Commons license, and indicate if changes were made.

Funded by SCOAP ${ }^{3}$.

\section{Appendix: Comparison with Powheg-Box}

To verify the Herwig 7 implementation of the NLO matching, we have also performed a comparison with the implementation of this process in the Powheg-Box framework [59]. For simplicity, we use the settings and cuts of Ref. [54] instead of our default setup here. The most important features of this are reproduced in the following: Protonproton collisions take place at a centre-of-mass energy of $8 \mathrm{TeV}$. As PDF, the MSTW2008nlo set [92] is used. 

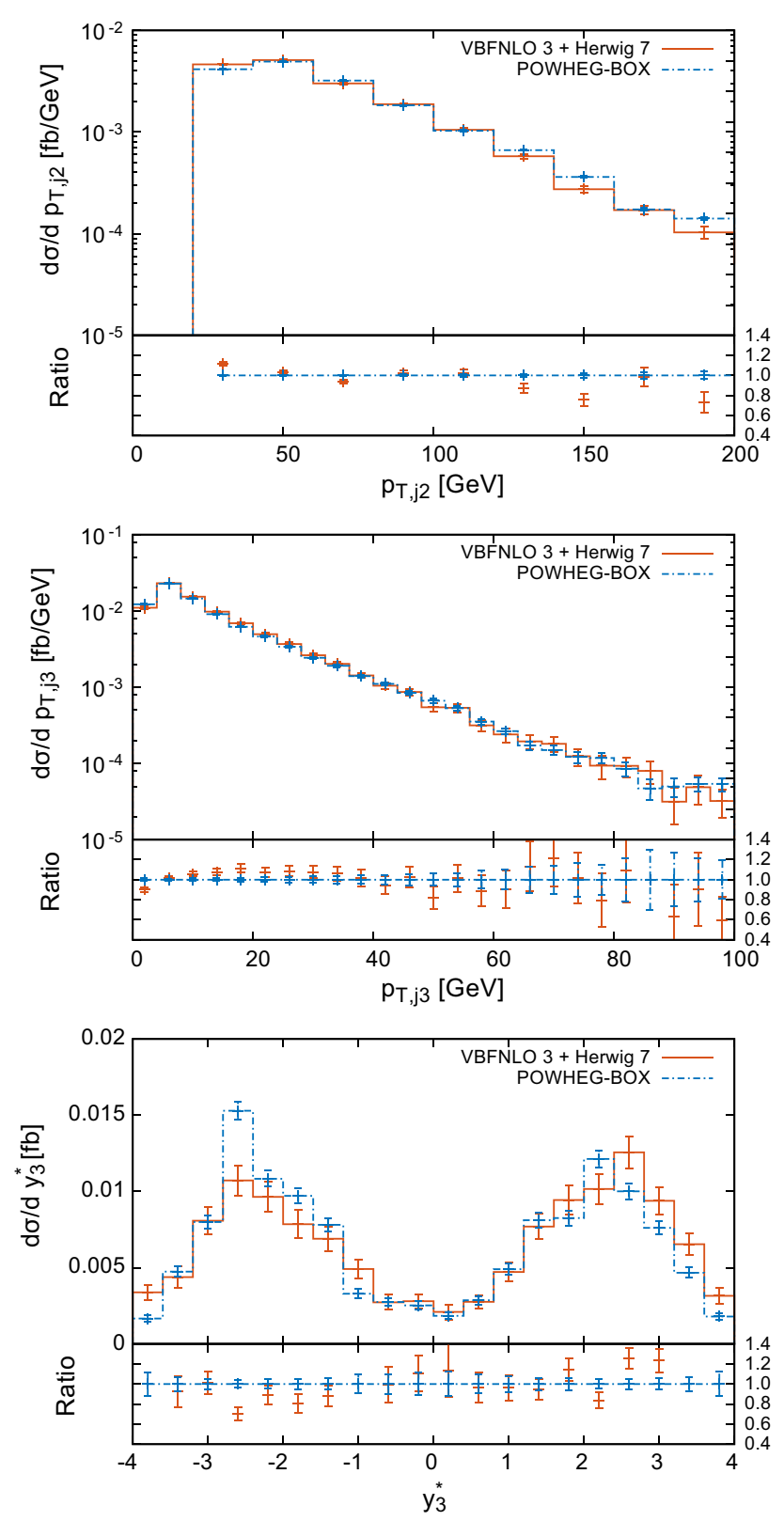

Fig. 10 Comparison of the multiplicative-matching implementations of Herwig 7 and POWHEG-BOX. Shown are distributions of the transverse momentum of the second (top) and third jet (centre) and the relative rapidity of the third leading jet with respect to the tagging jets (bottom). Cuts and settings follow Ref. [54]

Multiplicatively matched events at NLO are generated both with our VBFNLO $3+$ Herwig 7 setup and using thePowheg-Box implementation. These events are then fed into Pythia 6.4.25 [90] with the Perugia 0 tune, where the parton shower, hadronization and underlying event are added. Jets are clustered using the anti- $k_{T}$ algorithm with $R=0.4$, and at least two jets are required to be in the final state. As cuts we employ

$$
\begin{array}{rlrl}
p_{T, j} & >25 \mathrm{GeV}, & \left|y_{j}\right|<4.5, \\
p_{T, \ell 1} & >25 \mathrm{GeV}, & p_{T, \ell 2} & >15 \mathrm{GeV}, \\
\left|y_{\ell}\right| & <2.5, & p_{T, \text { miss }} & >25 \mathrm{GeV}, \\
R_{\ell \ell} & >0.3, & R_{j \ell} & >0.3 \\
m_{j 1, j 2} & >500 \mathrm{GeV}, & \left|y_{j 1}-y_{j 2}\right|>3.8, \\
y_{j 1} \times y_{j 2} & <0, & \left|\varphi_{\ell 1}-\varphi_{\ell 2}\right|<1.8, \\
& \min \left\{y_{j 1}, y_{j 2}\right\}<y_{\ell}<\max \left\{y_{j 1}, y_{j 2}\right\} . &
\end{array}
$$

In Fig. 10 we show the result of the comparison for three example distributions, namely the transverse momentum of the second and third jet and the rapidity of the third jet relative to the two tagging jets. Distributions involving the third jet are particularly sensitive to the matching procedure, so this constitutes an important cross-check, but we have checked other distributions as well. There is indeed good agreement between the two codes, with remaining differences stemming from subleading ambiguities in the algorithms such as cutoff choices and kinematic parametrizations. In particular also in the central region of the rapidity distribution the predictions agree excellently. For the two-jet inclusive cross section we obtain $338 \pm 3 \mathrm{ab}$ and $333.3 \pm 1.1 \mathrm{ab}$ and for the three-jet inclusive one $53.7 \pm 1.4 \mathrm{ab}$ and $52.0 \pm 0.4 \mathrm{ab}$ for VBFNLO 3+Herwig 7 and POWHEG-BOX, respectively.

\section{References}

1. R.N. Cahn, S. Dawson, Production of very massive Higgs Bosons. Phys. Lett. B 136, 196 (1984). [Erratum: Phys. Lett. B 138, 464(1984)]

2. S. Dawson, The effective W approximation. Nucl. Phys. B 249 , 42-60 (1985)

3. M.J. Duncan, G.L. Kane, W.W. Repko, W W physics at future colliders. Nucl. Phys. B 272, 517-559 (1986)

4. R.N. Cahn, S.D. Ellis, R. Kleiss, W.J. Stirling, Transverse momentum signatures for heavy Higgs bosons. Phys. Rev. D 35, 1626 (1987)

5. R. Kleiss, W.J. Stirling, Tagging the Higgs. Phys. Lett. B 200, $193-$ 199 (1988)

6. V.D. Barger, T. Han, R.J.N. Phillips, Improving the heavy Higgs boson two charged lepton-two neutrino signal. Phys. Rev. D 37, 2005-2008 (1988)

7. J.M. Butterworth, B.E. Cox, J.R. Forshaw, $W W$ scattering at the CERN LHC. Phys. Rev. D 65, 096014 (2002). [arXiv: hep-ph/0201098]

8. LHC Higgs Cross Section Working Group Collaboration, S. Dittmaier et al., Handbook of LHC Higgs Cross Sections: 1. Inclusive Observables. arXiv: 1101.0593

9. S. Dittmaier et al., Handbook of LHC Higgs Cross Sections: 2. Differential Distributions. arXiv: 1201.3084

10. LHC Higgs Cross Section Working Group Collaboration, J.R. Andersen et al., Handbook of LHC Higgs Cross Sections: 3. Higgs Properties. arXiv:1307.1347

11. D. Zeppenfeld, Collider physics, in Neutrinos in Physics and Astrophysics from 10**(-33) to $10^{* * 28} \mathrm{CM}$. Proceedings, Conference, TASI'98, Boulder, USA, June 1-26, 1998 (1999), pp. 303-350. arXiv: hep-ph/9902307 
12. A. Lazopoulos, K. Melnikov, F. Petriello, QCD corrections to tri-boson production. Phys. Rev. D 76, 014001 (2007). arXiv: hep-ph/0703273

13. V. Hankele, D. Zeppenfeld, QCD corrections to hadronic WWZ production with leptonic decays. Phys. Lett. B 661, 103-108 (2008). arXiv:0712.3544

14. F. Campanario, V. Hankele, C. Oleari, S. Prestel, D. Zeppenfeld, QCD corrections to charged triple vector boson production with leptonic decay. Phys. Rev. D 78, 094012 (2008). arXiv:0809.0790

15. T. Binoth, G. Ossola, C.G. Papadopoulos, R. Pittau, NLO QCD corrections to tri-boson production. JHEP 06, 082 (2008). arXiv:0804.0350

16. G. Bozzi, F. Campanario, V. Hankele, D. Zeppenfeld, NLO QCD corrections to $W^{+} W^{-} \gamma$ and $Z Z \gamma$ production with leptonic decays. Phys. Rev. D 81, 094030 (2010). arXiv:0911.0438

17. U. Baur, D. Wackeroth, M.M. Weber, Radiative corrections to W gamma gamma production at the LHC, PoS RADCOR2009, 067 (2010). arXiv:1001.2688

18. G. Bozzi, F. Campanario, M. Rauch, H. Rzehak, D. Zeppenfeld, NLO QCD corrections to $W^{ \pm} Z \gamma$ production with leptonic decays. Phys. Lett. B 696, 380-385 (2011). arXiv:1011.2206

19. G. Bozzi, F. Campanario, M. Rauch, D. Zeppenfeld, $W^{+-} \gamma \gamma$ production with leptonic decays at NLO QCD. Phys. Rev. D 83, 114035 (2011). arXiv: 1103.4613

20. G. Bozzi, F. Campanario, M. Rauch, D. Zeppenfeld, $Z \gamma \gamma$ production with leptonic decays and triple photon production at next-to-leading order QCD. Phys. Rev. D 84, 074028 (2011). arXiv: 1107.3149

21. B. Feigl, Electroweak Processes in the Standard Model and Beyond: Backgrounds to Higgs Physics and Semileptonic Decay Modes. PhD thesis, KIT, Karlsruhe, TP, 2013

22. T. Melia, K. Melnikov, R. Rontsch, G. Zanderighi, Next-to-leading order QCD predictions for $W^{+} W^{+} j j$ production at the LHC. JHEP 12, 053 (2010). arXiv:1007.5313

23. T. Melia, K. Melnikov, R. Rontsch, G. Zanderighi, NLO QCD corrections for $W^{+} W^{-}$pair production in association with two jets at hadron colliders. Phys. Rev. D 83, 114043 (2011). arXiv:1104.2327

24. T. Melia, P. Nason, R. Rontsch, G. Zanderighi, $W^{+} W^{+}$plus dijet production in the POWHEGBOX. Eur. Phys. J. C 71, 1670 (2011). arXiv: 1102.4846

25. B. Jäger, G. Zanderighi, NLO corrections to electroweak and QCD production of $W^{+} W^{+}$plus two jets in the POWHEGBOX. JHEP 11, 055 (2011). arXiv: 1108.0864

26. N. Greiner, G. Heinrich, P. Mastrolia, G. Ossola, T. Reiter, F. Tramontano, NLO QCD corrections to the production of $W^{+} W^{-}$ plus two jets at the LHC. Phys. Lett. B 713, 277-283 (2012). arXiv: 1202.6004

27. F. Campanario, M. Kerner, L. D. Ninh, D. Zeppenfeld, WZ production in association with two jets at next-to-leading order in QCD, Phys. Rev. Lett.111(5), 052003 (2013). arXiv:1305.1623

28. T. Gehrmann, N. Greiner, G. Heinrich, Precise QCD predictions for the production of a photon pair in association with two jets. Phys. Rev. Lett. 111, 222002 (2013). arXiv:1308.3660

29. F. Campanario, M. Kerner, L.D. Ninh, D. Zeppenfeld, Next-toleading order QCD corrections to $W^{+} W^{+}$and $W^{-} W^{-}$production in association with two jets, Phys. Rev. D 89(5), 054009 (2014). arXiv: 1311.6738

30. S. Badger, A. Guffanti, V. Yundin, Next-to-leading order QCD corrections to di-photon production in association with up to three jets at the Large Hadron Collider. JHEP 03, 122 (2014). arXiv: 1312.5927

31. F. Campanario, M. Kerner, L. D. Ninh, D. Zeppenfeld, Next-toleading order QCD corrections to $W \gamma$ production in association with two jets. Eur. Phys. J. C 74(5), 2882 (2014). arXiv:1402.0505

32. Z. Bern, L. J. Dixon, F. Febres Cordero, S. Hoeche, H. Ita, D.A. Kosower, N.A. Lo Presti, D. Maitre, Next-to-leading order $\gamma \gamma+2$ - jet production at the LHC, Phys. Rev. D 90(5), 054004 (2014). arXiv: 1402.4127

33. J. Alwall, R. Frederix, S. Frixione, V. Hirschi, F. Maltoni, O. Mattelaer, H.S. Shao, T. Stelzer, P. Torrielli, M. Zaro, The automated computation of tree-level and next-to-leading order differential cross sections, and their matching to parton shower simulations. JHEP 07, 079 (2014). arXiv:1405.0301

34. F. Campanario, M. Kerner, L.D. Ninh, D. Zeppenfeld, Next-toleading order QCD corrections to $\mathrm{ZZ}$ production in association with two jets. JHEP 07, 148 (2014). arXiv: 1405.3972

35. F. Campanario, M. Kerner, L. D. Ninh, D. Zeppenfeld, $Z \gamma$ production in association with two jets at next-to-leading order QCD. Eur. Phys. J. C 74(9), 3085 (2014). arXiv: 1407.7857

36. F. Campanario, M. Kerner, L.D. Ninh, M. Rauch, R. Roth, D. Zeppenfeld, NLO corrections to processes with electroweak bosons at hadron colliders. Nucl. Part. Phys. Proc. 261-262, 268-307 (2015)

37. D.L. Rainwater, R. Szalapski, D. Zeppenfeld, Probing color singlet exchange in $Z+$ two jet events at the CERN LHC. Phys. Rev. D 54, 6680-6689 (1996). arXiv: hep-ph/9605444

38. LHC Higgs Cross Section Working Group Collaboration, D. de Florian et al., Handbook of LHC Higgs Cross Sections: 4. Deciphering the Nature of the Higgs Sector. arXiv:1610.0792

39. T. Figy, C. Oleari, D. Zeppenfeld, Next-to-leading order jet distributions for Higgs boson production via weak boson fusion. Phys. Rev. D 68, 073005 (2003). arXiv: hep-ph/0306109

40. C. Oleari, D. Zeppenfeld, QCD corrections to electroweak $\ell v_{\ell} j j$ and $l^{+} l^{-} j j$ production. Phys. Rev. D 69, 093004 (2004). arXiv: hep-ph/0310156

41. B. Jäger, C. Oleari, D. Zeppenfeld, Next-to-leading order QCD corrections to $W^{+} W^{-}$production via vector-boson fusion. JHEP 07, 015 (2006). arXiv: hep-ph/0603177

42. B. Jäger, C. Oleari, D. Zeppenfeld, Next-to-leading order QCD corrections to $\mathrm{Z}$ boson pair production via vector-boson fusion. Phys. Rev. D 73, 113006 (2006). arXiv: hep-ph/0604200

43. G. Bozzi, B. Jäger, C. Oleari, D. Zeppenfeld, Next-to-leading order QCD corrections to $W^{+} Z$ and $W^{-} Z$ production via vector-boson fusion. Phys. Rev. D 75, 073004 (2007). arXiv: hep-ph/0701105

44. B. Jäger, C. Oleari, D. Zeppenfeld, Next-to-leading order QCD corrections to $W^{+} W^{+} j j$ and $W^{-} W^{-} j j$ production via weak-boson fusion. Phys. Rev. D 80, 034022 (2009). arXiv:0907.0580

45. A. Denner, L. Hosekova, S. Kallweit, NLO QCD corrections to $W^{+} W^{+} j j$ production in vector-boson fusion at the LHC. Phys. Rev. D 86, 114014 (2012). arXiv: 1209.2389

46. F. Campanario, N. Kaiser, D. Zeppenfeld, $\mathrm{W} \gamma$ production in vector boson fusion at NLO in QCD. Phys. Rev. D 89(1), 014009 (2014). arXiv: 1309.7259

47. K. Arnold et al., VBFNLO: A Parton level Monte Carlo for processes with electroweak bosons. Comput. Phys. Commun. 180, 1661-1670 (2009). arXiv:0811.4559

48. K. Arnold et al., VBFNLO: A Parton Level Monte Carlo for Processes with Electroweak Bosons - Manual for Version 2.5.0. arXiv: 1107.4038

49. K. Arnold et al., Release Note-Vbfnlo-2.6.0. arXiv:1207.4975

50. J. Baglio et al., Release Note-VBFNLO 2.7.0. arXiv:1404.3940

51. P. Nason, C. Oleari, NLO Higgs boson production via vector-boson fusion matched with shower in POWHEG. JHEP 02, 037 (2010). arXiv:0911.5299

52. B. Jäger, S. Schneider, G. Zanderighi, Next-to-leading order QCD corrections to electroweak $\mathrm{Zjj}$ production in the POWHEG BOX. JHEP 09, 083 (2012). arXiv:1207.2626

53. F. Schissler, D. Zeppenfeld, Parton shower effects on $W$ and $Z$ production via vector boson fusion at NLO QCD. JHEP 04, 057 (2013). arXiv:1302.2884

54. B. Jäger, G. Zanderighi, Electroweak $W^{+} W^{-} j j$ prodution at NLO in QCD matched with parton shower in the POWHEG-BOX. JHEP 04, 024 (2013). arXiv: 1301.1695 
55. B. Jäger, A. Karlberg, G. Zanderighi, Electroweak $Z Z j j$ production in the Standard Model and beyond in the POWHEG-BOX V2. JHEP 03, 141 (2014). arXiv:1312.3252

56. B. Jäger, F. Schissler, D. Zeppenfeld, Parton-shower effects on Higgs boson production via vector-boson fusion in association with three jets. JHEP 07, 125 (2014). arXiv: 1405.6950

57. P. Nason, A new method for combining NLO QCD with shower Monte Carlo algorithms. JHEP 11, 040 (2004). arXiv: hep-ph/0409146

58. S. Frixione, P. Nason, C. Oleari, Matching NLO QCD computations with Parton Shower simulations: the POWHEG method. JHEP 11, 070 (2007). arXiv:0709.2092

59. S. Alioli, P. Nason, C. Oleari, E. Re, A general framework for implementing NLO calculations in shower Monte Carlo programs: the POWHEG BOX. JHEP 06, 043 (2010). arXiv:1002.2581

60. M. Ciccolini, A. Denner, S. Dittmaier, Strong and electroweak corrections to the production of Higgs +2 jets via weak interactions at the LHC. Phys. Rev. Lett. 99, 161803 (2007). arXiv:0707.0381

61. M. Ciccolini, A. Denner, S. Dittmaier, Electroweak and QCD corrections to Higgs production via vector-boson fusion at the LHC. Phys. Rev. D 77, 013002 (2008). arXiv:0710.4749

62. T. Figy, S. Palmer, G. Weiglein, Higgs production via weak boson fusion in the standard model and the MSSM. JHEP 02, 105 (2012). arXiv: 1012.4789

63. T. Figy, V. Hankele, D. Zeppenfeld, Next-to-leading order QCD corrections to Higgs plus three jet production in vector-boson fusion. JHEP 02, 076 (2008). arXiv:0710.5621

64. F. Campanario, T.M. Figy, S. Plätzer, M. Sjödahl, Electroweak Higgs boson plus three jet production at next-to-leading-order QCD. Phys. Rev. Lett. 111(21), 211802 (2013). arXiv:1308.2932

65. P. Bolzoni, F. Maltoni, S.-O. Moch, M. Zaro, Higgs production via vector-boson fusion at NNLO in QCD. Phys. Rev. Lett. 105, 011801 (2010). arXiv: 1003.4451

66. P. Bolzoni, F. Maltoni, S.-O. Moch, M. Zaro, Vector boson fusion at NNLO in QCD: SM Higgs and beyond. Phys. Rev. D 85, 035002 (2012). arXiv:1109.3717

67. M. Cacciari, F. A. Dreyer, A. Karlberg, G. P. Salam, G. Zanderighi, Fully differential vector-boson-fusion Higgs production at nextto-next-to-leading order, Phys. Rev. Lett. 115(8), 082002 (2015). arXiv: 1506.0266

68. J. Bellm et al., Herwig 7.0/Herwig++ 3.0 release note. Eur. Phys. J. C 76(4), 196 (2016). arXiv: 1512.0117

69. https://herwig.hepforge.org/. Accessed 5 June 2016

70. M. Bähr et al., Herwig++ physics and manual. Eur. Phys. J. C 58, 639-707 (2008). arXiv:0803.0883

71. S. Gieseke, P. Stephens, B. Webber, New formalism for QCD parton showers. JHEP 12, 045 (2003). arXiv: hep-ph/0310083

72. S. Plätzer, S. Gieseke, Coherent parton showers with local recoils. JHEP 01, 024 (2011). arXiv:0909.5593

73. S. Plätzer, S. Gieseke, Dipole showers and automated NLO matching in Herwig++. Eur. Phys. J. C 72, 2187 (2012). arXiv:1109.6256
74. S. Catani, M.H. Seymour, A General algorithm for calculating jet cross-sections in NLO QCD, Nucl. Phys. B 485, 291-419 (1997). arXiv: hep-ph/9605323. [Erratum: Nucl. Phys. B 510, 503 (1998)]

75. S. Catani, S. Dittmaier, M.H. Seymour, Z. Trocsanyi, The Dipole formalism for next-to-leading order QCD calculations with massive partons. Nucl. Phys. B 627, 189-265 (2002). arXiv: hep-ph/0201036

76. https://www.itp.kit.edu/vbfnlo/. Accessed 5 June 2016

77. S. Alioli et al., Update of the Binoth Les Houches Accord for a standard interface between Monte Carlo tools and one-loop programs. Comput. Phys. Commun. 185, 560-571 (2014). arXiv: 1308.3462

78. A. Buckley, J. Butterworth, L. Lonnblad, D. Grellscheid, H. Hoeth, J. Monk, H. Schulz, F. Siegert, Rivet user manual. Comput. Phys. Commun. 184, 2803-2819 (2013). arXiv: 1003.0694

79. https://herwig.hepforge.org/plots/herwig7.0/. Accessed 5 June 2016

80. S. Plätzer, M. Rauch, et al. to be published tzer, M. Rauch, et al, Precision LHC event generation with matchbox (to be published)

81. S. Frixione, B.R. Webber, Matching NLO QCD computations and parton shower simulations. JHEP 06, 029 (2002). arXiv:hep-ph/0204244

82. M.H. Seymour, Matrix element corrections to parton shower algorithms. Comput. Phys. Commun. 90, 95-101 (1995). arXiv: hep-ph/9410414

83. G. Miu, T. Sjostrand, $W$ production in an improved parton shower approach. Phys. Lett. B 449, 313-320 (1999). arXiv: hep-ph/9812455

84. S. Plätzer, ExSample: a library for sampling sudakov-type distributions. Eur. Phys. J. C 72, 1929 (2012). arXiv:1108.6182

85. J. Bellm, G. Nail, S. Plätzer, P. Schichtel, A. Siödmok, Parton shower uncertainties with Herwig 7: benchmarks at leading order, Eur. Phys. J. C 76(12), 665 (2016). arXiv:1605.0133

86. L.A. Harland-Lang, A.D. Martin, P. Motylinski, R. S. Thorne, Parton distributions in the LHC era: MMHT 2014 PDFs. Eur. Phys. J. C 75(5), 204 (2015). arXiv: 1412.3989

87. M. Cacciari, G.P. Salam, G. Soyez, The Anti-k(t) jet clustering algorithm. JHEP 04, 063 (2008). arXiv:0802.1189

88. ATLAS Collaboration, G. Aad et al., Observation and measurement of Higgs boson decays to WW ${ }^{*}$ with the ATLAS detector. Phys. Rev. D 92(1), 012006 (2015). arXiv:1412.2641

89. C.M.S. Collaboration, S. Chatrchyan et al., Measurement of Higgs boson production and properties in the WW decay channel with leptonic final states. JHEP 01, 096 (2014). arXiv:1312.1129

90. T. Sjostrand, S. Mrenna, and P. Z. Skands, PYTHIA 6.4 Physics and Manual. JHEP05, 026 (2006). arXiv: hep-ph/0603175

91. F. Schissler, Parton Shower Corrections to Vector-Boson Fusion Processes at the Large Hadron Collider in Next-to-Leading Order QCD Precision. $\mathrm{PhD}$ thesis, KIT, Karlsruhe, 2014

92. A.D. Martin, W.J. Stirling, R.S. Thorne, G. Watt, Parton distributions for the LHC. Eur. Phys. J. C 63, 189-285 (2009). arXiv:0901.0002 